\title{
EFECTIVIDAD DE LA INTERVENCIÓN PSICOSOCIAL EN EL ALIVIO DEL SU- FRIMIENTO DE PERSONAS CON ENFERMEDADES AVANZADAS
}

Autor: Dolors Mateo Ortega

Directores de Tesis: Dr. Joaquín T. Limonero García y Dr. Xavier Gómez BatisteAlentorn

Centro: Universitat Autònoma de Barcelona. Facultad de Psicología. Departamento de Psicologia Básica, Evolutiva y de la Educación

Fecha de lectura: 4 de marzo de 2016

E-mail: dmateo@iconcologia.net

El principal objetivo de esta tesis consiste en demostrar que las intervenciones psicosociales específicas mejoran el sufrimiento y el malestar de los pacientes en situación de final de vida que reciben cuidados paliativos, especialmente en aquellos que tienen un alto nivel de sufrimiento o malestar emocional.

Para ello se ha analizado la evolución de parámetros psicológicos y espirituales de 8333 pacientes, visitados por 137 profesionales de 29 equipos de atención psicosocial en un estudio de diseño cuasi-experimental, prospectivo, multcéntrico de un solo grupo pre-test post-test.

Se ha analizado la evolución del estado de ánimo, la ansiedad, el malestar emocional, el sentido de la vida, la sensación de paz y perdón, y el sufrimiento referido por el paciente en las visitas realizadas por el equipo de atención psicosocial así como la valoración global del estado del paciente por parte del profesional.

Así mismo, se ha analizado la evolución de estos parámetros en función de la complejidad que presentaba el paciente en la primera visita, considerándose complejos aquellos pacientes que en la visita inicial presentaban elevados niveles de ansiedad, malestar y estado de ánimo, que a pesar de tener creencias espirituales valoraba que no le ayudaban en la situación actual, y que percibían el paso del tiempo como lento además de tener una valoración global parte del profesional de un bajo nivel de adaptación.

Los resultados obtenidos nos permiten afirmar que la intervención psicosocial tiene un efecto positivo en la reducción de los parámetros psicológicos y espirituales evaluados, demostrándose eficaz en el contexto de la atención a personas con enfermedades avanzadas que reciben cuidados paliativos.

Asimismo, se puede afirmar que estas intervenciones son más efectivas en aquellos pacientes con un sufrimiento muy elevado, confirmando así que tal y como recomiendan la mayoría de sociedades científicas, hay un grupo de pacientes con una complejidad mayor que requieren de intervenciones de profesionales del ámbito psicosocial. El nivel de sufrimiento en éste grupo de pacientes parte de un nivel más elevado que el de los pacientes sin criterios de complejidad en la visita ba- 
sal, y tras las sucesivas intervenciones psicosociales va disminuyendo hasta situarse prácticamente al mismo nivel que el de los pacientes no complejos.

Por último nos parece importante remarcar la necesidad de atender a la globalidad de las necesidades de la persona, abarcando tanto aspectos de naturaleza psicológica como social, englobando en ellas a los aspectos emocionales y a las necesidades espirituales de los pacientes.

Palabras clave: intervención psicosocial, atención integral, efectividad, final de vida, cuidados paliativos, multicéntrico, complejidad psicosocial. 